\title{
Updates in Genetic Molecular Targeted Therapy for Glioblastoma
}

\author{
Bader A. Abdelmaksoud \\ Department of Clinical Oncology and Nuclear Medicine, Faculty of Medicine, Zagazig University, Egypt
}

Copyright $(2016$ by authors, all rights reserved. Authors agree that this article remains permanently open access under the terms of the Creative Commons Attribution License 4.0 International License

\begin{abstract}
Glioblastoma multiforme (GBM) is the most common and devastating primary brain tumor in adults. Current standard treatment after maximal safe surgical resection with concurrent chemoradiotherapy by temozolomide and radiation therapy has a modest improvement in progression free and overall survival with frequent recurrences. With advancement in molecular biology and gene technology a novel treatment strategies with promising outcome have been provided. In this review, the most commonly studied molecular targets in the treatment of glioblastoma will be discussed. EGFR and its variant EGFRvIII have an important role in GBM cellular proliferation. VEGF and its receptor are active factors in tumor angiogenesis. Ras pathway, its regulators and others as protein kinase $\mathrm{C}$ and integrin contribute to tumorigenesis and resistance to conventional therapy. With inhibition of the aforementioned pathways more direct and targeted method of GBM treatment will be provided. Also combination of these treatment modalities may create an innovative therapeutic approach for GBM management.
\end{abstract}

Keywords Glioblastoma, Targeted Therapy, Molecular Biology, EGFR, EGFRvIII

\section{Introduction}

Glioblastoma multiforme (GBM or grade IV glioma) is the most common primary malignant brain tumor and account for $(45.6 \%)$ of brain malignancy. It is a diffusely infiltrative and usually recurrent brain tumor. The prognosis of glioblastoma is poor with median survival of 1 - 2 years with conventional treatment $[1,2,3]$.

Molecular subtypes of GBM were defined previously with beginning of the distinction of primary (also known as de novo) and secondary GBM based on presence or absence of a precursor lesion. Primary glioblastoma is the most common type $(\sim 90 \%)$, it arises de novo, without evidence of a precursor lesion and it is common in older adults. Secondary glioblastoma progressed from pre-existent lesions such as other astrocytomas (WHO grades II or III) $[4,5]$.

Gene expression profile studies in GBM have revealed several transcriptional subclasses with distinct molecular characteristics. The proneural subtype is common in young adults, usually corresponds to the secondary glioblastoma, has neuronal differentiation and usually associated with better outcome, characterized by IDH/TP53 mutations and normal EGFR/PTEN/Notch signaling. On the other hand mesenchymal GBM displays high expression of CHI3L1, MET and genes involved in the tumor necrosis factor (TNF) and nuclear factor of $\kappa$-light polypeptide gene enhancer in B-cells (NFKB) pathways and high frequency of neurofibromatosis type 1 mutation/deletion, common in older adults and has poor outcome [6-9]. Classical subtype displays epidermal growth factor receptor (EGFR) amplification or mutations, chromosome 7 amplifications, chromosome 10 deletions and Ink4a/ARF locus deletion. Neural and pediatric subtypes have particular molecular abnormalities where pediatric one characterized by frequent PDGFR amplification, frequent gains of chromosome 1q, lack IDH mutations and lower frequencies of chromosome 7 gain and 10q loss [10]. Another finding was described to GBM pathogenesis describing the FGFR-TACC fusion in a small fraction of glioblastoma. Chromosomal translocations cause in-frame fusion of the tyrosine kinase domains of FGFR and TACC. The abnormal protein produced localizes to the mitotic spindle and induces chromosomal instability and aneuploidy promoting oncogenesis [11].

In this review, up-to-date information on the molecular targets and their inhibitors will be provided. Currently, these agents are in different stages of clinical trials. The goal is to stimulate the development of new treatment strategies that can improve the outcome of this disease with poor prognosis. As known, multiple signaling pathways are deregulated, so the use of combination drugs along with personalized targeting approach would provide better prognosis in the future. 


\section{Molecular Targets and Their Inhibitors}

Glioblastoma is considered to be one of the most molecularly characterized human cancers. Molecular profiling studies have resulted in the identification of molecular prognostic targets as well as identified molecular inhibitors that could be potentially used in the development of novel treatments in glioblastoma.

\subsection{Growth Factors Receptors Inhibitors}

Growth factors receptors inhibitors are bioactive, usually aromatic, small molecules that can bind to active site of a receptor tyrosine kinase (RTK), thus preventing phosphorylation resulting in inhibition, regulation, or modulation of signaling pathways. Some potent receptor tyrosine kinase inhibitors will exhibit selectivity for a certain RTK, while others are less selective [24]. Below, RTKIs used in treat of glioblastoma will be identified. There are 3 categories based on the primary RTK target: epidermal growth factor receptor (EGFR), vascular endothelial growth factor receptor (VEGFR), and platelet derived growth factor receptor (PDGFR).

\section{Epidermal Growth Factor Receptor Inhibitors}

EGFR is amplified in $\sim 40 \%$ of primary GBM and its overexpression usually absent in secondary GBM; it is a mutually exclusive finding relative to IDH1/2 mutations [12-14]. In GBM, activation of EGFR is associated with gain of function missense mutations or in-frame deletions affecting the extracellular domain. Approximately $50 \%$ of EGFR-amplified GBM express EGFRvIII(variant III), a constitutively active, ligand-independent mutant receptor with impaired downregulation [15-18].

Also activation of EGFR occurs by receptor overexpression associated with multiple ligand-dependent and independent mechanisms in glioblastoma, with generation of intracellular mitogen-activated protein kinase (MAPK), phosphatidylinositol-3- OH kinase (PI3K), Src kinase pathway signaling and STAT transcription factor activation [19].

A variety of studies for EGFR inhibitors have been and continue to be evaluated for GBM including EGFR TKIs, nonradiolabeled MAb (unconjugated) and radiolabeled monoclonal antibodies (conjugated) where fragment toxin conjugates are administered either systemically or locally [20-23].

EGFR TKIs are classified as first generation reversible inhibitors that target EGFR and its co-receptor HER2 (gefitinib, erlotinib, and lapatinib), second generation irreversible inhibitors (afatinib, dacomitinib, and neratinib) and third generation inhibitors (AZD9291 and CLO-1686) targeting the $\mathrm{T} 790 \mathrm{M}$ mutation associated with acquired resistance to first generation EGFR TKIs in non-small cell lung cancer. In addition, there are multitargeting TKIs with inhibitory capability against EGFR, as well as other growth factors, that have been evaluated for GBM such as vandetanib and AAE788, both of which block EGFR and VEGFR [24-26].

Monoclonal antibodies either conjugated or unconjugated have been developed to target both EGFR and EGFRvIII, the most of these agents with promising results are cetuximab, panitumumab and nimotuzumab. The unconjugated antibodies bind the extracellular domain of EGFR, and they are also suggested to cause internalization of EGFRvIII, though clinical trials have had varying results of these agents $[27,28]$.

Herein, this review will cover as most as possible the clinical studies and ongoing trials to determine the role EGFR inhibitors in treatment of glioblastoma and find the future hope to improve the response rate and survival of this fatal disease.

\section{1- Epidermal Growth Factor TKIs}

Results of pre-clinical studies demonstrate the efficacy of TKIs to inhibit tumor growth, angiogenesis, proliferation and survival in several different EGFR transfected GBM cell lines. However, these results showed modest response rates in GBM patients [29-33]. Table-1 shows different EGFR TKIs and their studies.

Geftinib was the first EGFR inhibitor tested in recurrent glioblastoma [52]. Although gefitinib has a limited BBB permeability, with increasing the dose there is acceleration of passive permeability [53]. The first open-label, single-center phase II trial was conducted on fifty-seven patients with first recurrence of glioblastoma, open biopsy or resection was undertaken for confirmation of tumor recurrence. Each patient initially received $500 \mathrm{mg}$ of gefitinib, orally, once daily, dose escalation to $750 \mathrm{mg}$ then $1,000 \mathrm{mg}$ was given, the final conclusion showed that gefitinib was well tolerated and had activity in patients with recurrent glioblastoma [34]. The next studies were conducted to evaluate the role of gefitinib in combination with other agents. Reardon and collegues conducted a phase I study to determine the maximum tolerated dose and dose-limiting toxicity of gefitinib plus sirolimus, an inhibitor of the mammalian target of rapamycin, among patients with recurrent malignant glioma, thirty-four patients with progressive disease after prior radiation therapy and chemotherapy were enrolled and the study showed that gefitinib plus sirolimus can be safely co administered on a continuous daily dosing schedule, and established the recommended dose level of these agents. Subsequent studies of gefitinib combinations were evaluated with temozolamide and concurrently with radiation therapy [35-39]. Currently there are no ongoing trials to evaluate the role of gefitinib in treatment of glioblastoma. 
Table 1. EGFR TKIs and their studies in GBM

\begin{tabular}{|c|c|c|}
\hline Agent & studies & Other ongoing trials \\
\hline 1-Gefitinib & $\begin{array}{c}\text { Richetal(2004)[34] } \\
\text { Reardonetal(2006)[35] } \\
\text { Pradosetal(2008)[36] } \\
\text { Hegietal(2011)[37] } \\
\text { Uhmetal(2011)[38] } \\
\text { Chakravartietal(2013)[39] }\end{array}$ & None \\
\hline 2- Erlotinib & $\begin{array}{c}\text { Pradosetal(2006)[40] } \\
\text { Krishnanetal(2006)[41] } \\
\text { Brownetal(2008)[42] } \\
\text { Pradosetal(2009)[43] } \\
\text { Peereboometal(2010)[44] } \\
\text { Reardonetal(2010)[45] } \\
\text { Raizeretal(2010)[46] } \\
\text { VandenBentetal(2010)[47] } \\
\text { Yungetal(2010)[48] } \\
\text { Wenetal(2014)[49] }\end{array}$ & $\begin{array}{c}\text { NCT01110876 (terminated due to anticipated } \\
\text { toxicity) } \\
\text { NCT00301418 (completed and waiting for } \\
\text { results) }\end{array}$ \\
\hline 3- Afatinb & $\begin{array}{l}\text { Eisenstatetal(2010)[50] } \\
\text { Reardonetal(2014)[51] }\end{array}$ & None \\
\hline 4- Lpatinib & $\begin{array}{l}\text { Thiessenetal(2010)[55] } \\
\text { Reardonetal(2012)[56] }\end{array}$ & $\begin{array}{l}\text { NCT01591577 (currently ongoing and } \\
\text { recruiting participant) }\end{array}$ \\
\hline 5- Dacomitinib & None & $\begin{array}{l}\text { NCT01520870 (currently ongoing but not } \\
\text { recruiting participant) } \\
\text { NCT01112527(currently ongoing but not } \\
\text { recruiting participant) }\end{array}$ \\
\hline 6-Neratinib & None & $\begin{array}{l}\text { NCT01953926(currently ongoing and } \\
\text { recruiting participant) }\end{array}$ \\
\hline
\end{tabular}

Phase II studies 'were conducted to evaluate the role of erlotinib for newly diagnosed GBM patients. Median overall survival did not correlate with the presence of EGFRvIII, EGFR amplification, or PTEN loss in the 97 patients treated on NCCTG NO177, a single-arm phase I/II study [42]. Another additional study with somewhat discrepant results was conducted in single-institution settings, where median OS in 27 patients was only 8.6 months [44]. Combination of erlotinib with temozolomide and radiotherapy was evaluated in phase II open-label, prospective, single-arm study, median survival was 19.3 months compared to 14.1 months in the combined historical control studies and the author concluded that combination of erlotinib and temozolomide during and following radiotherapy had better survival than historical controls [43]. For recurrent or relapsed GBM erlotinib was evaluated extensively. Van den Bent and colleagues conducted a randomized, controlled, phase II trial on 110 patients with progressive GBM after prior radiotherapy, the patients were randomly assigned to either erlotinib or a control arm that received treatment with either temozolomide or carmustine (BCNU), the primary end point was 6-month progression free survival (PFS). Tumor specimens obtained at first surgery were investigated for EGFR expression, EGFRvIII mutants, EGFR amplification, EGFR mutations in exons 18, 19, and 21; and pAkt [47].
Although the treatment was tolerated the author concluded that erlotinib has insufficient single-agent activity in unselected GBM. Another phase II open labeled study by Yung et al evaluating erlotinib efficacy and safety in patients with first-relapse glioblastoma and assessed whether response was related to EGFR amplification and/or concomitant use of enzyme-inducing antiepileptic drugs (EIAEDs), erlotinib was taken daily until progression. Starting dose was $150 \mathrm{mg}$ for patients not taking EIAEDs and $300 \mathrm{mg}$ for patients taking EIAEDs. The results were acceptable to the author despite limited number of responses; 6-month PFS and median survival reached or exceeded the previously reported values for patients undergoing chemotherapy for recurrent glioblastoma. EGFR amplification was not associated with erlotinib activity [48]. In phase I/II study of the EGFR inhibitor erlotinib (150 $\mathrm{mg} /$ day) and the mTOR inhibitor temsirolimus, patients were firstly received temsirolimus $50 \mathrm{mg}$ weekly, then with dose adjusted on toxicities. In the phase II component, the primary endpoint was 6-month progression-free survival (PFS6) among glioblastoma patients, of 42 evaluated glioblastoma patients, 12 (29\%) achieved stable disease and 6 months PFS was $13 \%$. Among 16 anaplastic glioma patients, $1(6 \%)$ achieved complete response, $1(6 \%)$ partial response and 2 $(12.5 \%)$ stable disease, with 6 months PFS of $8 \%$. But 
because of increased toxicity, the maximum tolerated dosage of temsirolimus in combination with erlotinib required de-escalation. Also presence of EGFR variant III, phospho-EGFR, and EGFR amplification did not correlate with survival [49].

Currently there are two ongoing trials evaluating the role of erlotinib in treatment of glioblastoma, NCT00301418 is an open labeled non randomized single group study evaluating safety and efficacy of erlotinib for treatment of relapsed/refractory glioblastoma multiforme and anaplastic astrocytoma, this trial was completed and waited for results. NCT01110876 is Phase I / II adaptive randomized trial of vorinostat, erlotinib and temozolomide in adults With recurrent glioblastoma multiforme to determine the maximum tolerated dose (MTD) of vorinostat + erlotinib versus vorinostat + erlotinib + temozolomide in adult patients with recurrent glioblastoma multiforme (GBM) and anaplastic gliomas and to evaluat the efficacy of vorinostat + erlotinib versus vorinostat + erlotinib + temozolomide in patients with recurrent glioblastoma multiforme as progression free survival using a two arm adaptive randomization phase II trial design.

Afatinib, a second-generation/irreversible EGFR TKIs has been evaluated in GBM, a phase II study of afatinib with or without temozolomide was undertaken to evaluate efficacy and safety of afatinib and afatinib with temozolomide compared to temozolomide alone in recurrent GBM and to assess molecular determinants of response to afatinib. The results of this study were statistically worse for afatinib compared to temozolomide and afatinib with temozolomide to temozolomide alone. But preliminary biomarker data in 54 patients suggest that EGFRvIII-positive patients treated with afatinib/afatinib with temozolomid showed durable disease control. The final conclusion was that afatinib has limited single-agent activity in recurrent GBM [50]. Recent phase I/II trial has been conducted to evaluate the maximum tolerated dose and pharmacokinetics of afatinib plus temozolomide and the efficacy and safety of afatinib alone compared to temozolomide alone in patients with recurrent glioblastoma (GBM). The findings were that the dose of afatinib was $40 \mathrm{mg} /$ day with temozolomode based on safety data from phase I. Most observed adverse events in phase II were diarrhea and rash, also co-administration of afatinib and temozolomide did not affect pharmacokinetics. 6 months PFS rate was $3 \%$ with afatinib, $10 \%$ with afatinib and temozolomide, and $23 \%$ for temozolomide alone. Participants with EFGRvIII-positive tumors had Median PFS longer than those with EGFRvIII-negative tumors in afatinib treated group. The author finally concluded that despite afatinib has a manageable safety profile it has a limited single-agent activity in unselected recurrent GBM patients [51].

Lapatinib is a small molecule TKI that dually targets EGFR1, 2. Lapatinib enters the cell and binds to intracellular domain of tyrosine kinase receptor, resulting in complete blockade of autophosphorylation reaction and complete cessation of downstream cascade [54]. Although this hopeful mechanism of action, clinical studies of lapatinib for glioblastoma until now are few. A phase I/II study lapatinib was undertaken for patients with recurrent glioblastoma multiforme to determine response rate, pharmacokinetics, recommended dose in patients taking enzyme-inducing anti-epileptic drugs (EIAEDs) and to explore relationship of molecular genetics to outcome. In this study, the patients were subjected to lapatinib $1,000 \mathrm{mg}$ PO bid, escalation was continued if there was no dose-limiting toxicity. Patients not on EIAEDs enrolled in the phase II arm (lapatinib $750 \mathrm{mg}$ bid po). PTEN and EGFRvIII status were determined by immunohistochemical and quantitative RT PCR studies respectively. Although treatment was tolerated, accrual ceased because of no responses and the author concluded that lapatinib did not show significant activity in patients with glioblastoma [55]. Another phase II study was conducted to evaluate the antitumor activity of lapatinib $1,000 \mathrm{mg} / \mathrm{d}$ in combination with pazopanib $400 \mathrm{mg} / \mathrm{d}$ for patients with GBM with known PTEN/EGFRvIII status not receiving enzyme-inducing anticonvulsants (EIACs). Also, although treatment protocol was safe the response rate was low and the author recommended for evaluation of intratumoral drug delivery and activity for hypothesis-testing trials with targeted agents in malignant glioma [56].

Although the previous studies showed no promising results of lapatinib for glioblastoma, there is ongoing Phase II trial testing pulse dosing of lapatinib combined with temozolomide and regional radiation therapy as an upfront treatment of patients with newly-diagnosed GBM. The aim of this study is to evaluate the safety and efficacy of combination of Lapatinib with standard concurrent chemo-radiotherapy in patients with newly diagnosed glioblastoma multiforme. The expecting findings of this trial will answer if lapatinib when given twice weekly with higher doses during standard concurrent chem.-radiotherapy is safe or not, what are adverse effects of lapatinib in this protocol, what is the efficacy of labatinib in this protocol and whether the presence of genetic alterations specific proteins in the tumor samples will predict the efficacy of labatinib on the tumor. This study is currently recruiting participants (https://clinicaltrials.gov/ct2/show/NCT01591577).

Dacomitinib (PF299804), a recently introduced second generation irreversible EGFR Inhibitor with expected activity against EGFR and EGFRvIII positive glioblastom has been tested in preclinical phases to confirm its antitumor efficacy [57]. The results of this testing confirm that dacomitinib showes an effect on cell viability, self-renewal and proliferation in EGFR-amplified \pm EGFRvIII glioblastoma cells and will help to select molecular determinants of this response and to execute future trials with dacomitinib in newly diagnosed glioblastoma.

Currently there are two ongoing clinical trials to determine the effective role of dacomitinib in treatment of glioblastoma. NCT01112527 is an open-label, phase II trial of orally administered dacomitinib in adult patients with relapsed/recurrent glioblastoma to assess progression-free 
survival (efficacy) in patients with recurrent GBM and with EGFR amplification as well as evaluation of safety and tolerability of this agent. NCT01520870 is phase II pilot, prospective, open label, multicenter clinical trial, to evaluate the safety and efficacy of dacomitinib, a pan-HER irreversible inhibitor, in patients with recurrent glioblastoma with EGFR amplification or presence of EGFRvIII mutation, the primary end point is progression free survival and secondary end points are safety, overall survival, and others (https://clinicaltrials.gov/ct2/show/NCT01520870).

Neratinib (HKI-272) is a second generation irreversible pan-EGFR TKI was three-fold to four-fold more effective at suppressing cell proliferation than first generation drugs. Neratinib was studied in treatment of non-small cell lung cancer and breast cancer [58, 59]. Currently there is an open-label, multicenter, multinational, phase II study evaluating the efficacy and safety of neratinib in patients with solid tumors with activating HER2, HER3 or EGFR mutations or with EGFR gene amplification.

As shown previously, EGFR TKIs have the ability to inhibit tumor cell growth, angiogenesis, survival, and proliferation in several different EGFR transfected glioblastoma cell lines. Also second generation TKIs with its irreversible mechanism of action will have promise in future. But as response rates in GBM patients are disappointing for many inhibitors these results do not appear to be clinically translatable [29]. So further researches should be conducted to follow the efficacy of these agents and to explore the limitations of these drugs in treatment of this fatal disease and test its combination with other available anticancer drugs.

\section{2-Monoclonal Antibodies (MABs) Targeting EGFR}

EGFR was the first molecular target attacked by monoclonal antibodies and applied for cancer therapy [60]. In glioblastoma, monoclonal antibodies both unconjugated and conjugated directed towards wild EGFR and mutated EGFR have been developed for therapeutic use [61]. In this review different EGFR MABs will be represented with respect to its role in treatment of GBM. Table 2 shows different EGFR MABs studies and their future directions.

Table 2. MABs targeting EGFR in glioblastoma

\begin{tabular}{|c|c|c|}
\hline Agent & Studies & Other ongoing studies \\
\hline Cetuximab & $\begin{array}{c}\text { Combsetal(2006)[62] } \\
\text { Neynsetal(2009)[63] } \\
\text { Hasselbalchetal(2010)[64] } \\
\text { Lvetal(2012)[65] } \\
\text { Mortensenetal(2013)[66] }\end{array}$ & $\begin{array}{l}\text { NCT00311857 } \\
\text { NCT01238237 }\end{array}$ \\
\hline Nimotuzumab & $\begin{array}{c}\text { Diazetal(2012)[67] } \\
\text { Hongetal(2012)[68] } \\
\text { Solomónetal(2013)[69] } \\
\text { Wangetal(2014)[70] } \\
\text { Solomonetal(2014)[71] } \\
\text { Westphaletal(2012)[72] }\end{array}$ & NCT00753246 \\
\hline Panitumimab & Non & NCT01017653 \\
\hline Mab 425 & Lietal(2010)[73] & NCT01317888 \\
\hline MAb806 & Johnsetal(2010)[74] & Non \\
\hline ABT 414 & Non & $\begin{array}{c}\text { NCT01800695 } \\
\text { DRUG M12-356 }\end{array}$ \\
\hline AMG595 & Hamblettetal(2015)[75] & NCT01475006 \\
\hline MR1-1 & $\begin{array}{l}\text { Dingetal(2010)[76] } \\
\text { Choietal(2013)[77] }\end{array}$ & NCT01009866 \\
\hline
\end{tabular}


Cetuximab is a monoclonal antibody targeting extra cellular domain of the EGFR. Cetuximab shows activity against different tumor types, either alone or in combination with chemotherapy and/or radiation therapy [78]. In GBM, cetuximab is more efficient in inhibiting the EGFRvIII compared to other small molecule EGFR inhibitors [79]. Phase II study by Neyns and colleagues was undertaken, in which two-arm, open-label, phase II study in which the patients were stratified according to their epidermal growth factor receptor (EGFR) gene amplification status. The results were that there was insignificant correlation between response, survival and EGFR amplification and the author concluded that, although treatment was tolerated cetuximab had limited activity in patients with progressive high grade glioma [63]. Another study was conducted to evaluate safety and efficacy of cetuximab when combined with bevacizumab and irinotecan in patients with recurrent glioblatoma, the results were acceptable as regard to radiologic response, 6 months progression free survival and overall survival with some toxicity profiles. According to this study combination of cetuximab with bevacizumab and irinotecan in patient with recurrent glioblastoma was well tolerated except for skin toxicity, with an encouraging response rate. As regard to the efficacy, the results did not seem to be superior compared to that with bevacizumab and irinotecan alone [64].

Nimotuzumab is a humanized monoclonal antibody targeting EGFR and clinical studies are ongoing globally to evaluate it in different indications [80]. Preclinical studies to evaluate the efficacy of nimotuzumab as a single agent therapy in human glioblastoma were conducted. Diaz and colleagues conducted preclinical study to examine the antitumor activity of three different single therapies in nude mice bearing both subcutaneous and orthotopic brain xenografts of the U87MG human glioblastoma cell lines. According to the results obtained, the author concluded that nimotuzumab had potential efficacy as a single agent against EGFR-amplified human glioblastoma [67]. A phase I/II clinical trial was undertaken to determine the role of nimotuzumab when combined with chemoradiotherapy in patients with glioblastoma. The results of this study were promising and the final conclusion was that addition of nimotuzumab to standered concurrent chemo radiotherapy was effective for malignant gliomas [68]. Another randomized, double blind, multicentric clinical trial was conducted for patients with high grade gliomas (41 anaplastic astrocytoma and 29 glioblastoma multiforme) that received radiotherapy plus nimotuzumab or placebo. The results of this trial were very accepted by the investigators and they concluded that nimotuzumab showed excellent therapeutic profile and significant survival benefit when combined with irradiation [69]. Subsequently the investigator in previous study underwent another single institution experience study of nearly the same protocol to determine the role of combination of nimotuzumab and radiotherapy for high grade gliomas. The results were comparable to the previous trials and the author confirmed that nimotuzumab was a very well-tolerated drug, lacking cumulative toxicity after maintenance doses and these results validated the previous data of survival gain when nimotuzumab combined with radiotherapy in patients with newly diagnosed high-grade gliomas [71]. Combination of nimotuzumab with standard concurrent chemradiothery was evaluated for safety and efficacy in treatment of gliobalstoma patients with favorable results as regards to overall and progression free survival and author concluded that nimotuzumab showed favorable safety and tolerability profiles in newly diagnosed glioblastom in Chinese patients when combined with standard concurrent chemo-radiotherapy [70]. An open label, randomized, multicenter Phase III trial of combination of nimotuzumab with standard concurrent chemo-radiotherapy was conducted for patients with histologically confirmed, newly diagnosed GBM [72] the final results of this trial showed that nimotuzumab was safe and did not show the severe toxicity when added to standard therapy and the author recommend for future studies to detect efficacy of nimotuzumab focusing on patients with nonmethylated MGMT and EGFR-positive GBM.

Studies of conjugated MABs were conducted to determine the efficacy of radio immunotherapy of radioactive labeled anti-epidermal growth factor receptor monoclonal antibody in patients with newly diagnosed GBM. A single-institution phase II study was undertaken to evaluate efficacy of adjuvant radio immunotherapy with I (125) -labeled anti-epidermal growth factor receptor 425 murine monoclonal antibody (MAB 425) in patients with newly diagnosed GBM, a total of 192 patients with glioblastoma were treated with I (125) -mAb 425 over a course of intravenous injections of $1.8 \mathrm{GBq}$ following surgery and radiation therapy for 3 week with or without temozolomide, treatment was safe and well tolerated [73]. NCT01317888 is an ongoing trial to allow patients with brain tumors who had previously received I (125) -MAB 425 to receive additional course(s) of that agent again until their brain tumor begins to grow, developing side effects from the treatment, or changes of their medical condition (e.g. pregnancy, infection with human immunodeficiency virus (HIV) or development of another cancer). ABT-414, a novel anti-EGFR monoclonal antibody drug conjugate is currently being evaluated in a Phase I trial with concurrent radiotherapy and temozolomide in patients with newly diagnosed glioblastoma. NCT01800695 is an open labeled nonrandomized Phase I study evaluating the safety and pharmacokinetics of ABT-414 for patients with Glioblastoma.

Drug M12-356 is another ongoing phase I study evaluating the safety and pharmacokinetics of ABT-414 in combination of radiation with temozolomide or temozolomide alone for patients with GBM.

AMG 595 is an antibody drug conjugate, highly selective anti-EGFRvIII was developed to treat patients with EGFRvIII-positive glioblastoma. This was presented by Hamblett et al who incubated U251 cells expressing EGFRvIII with AMG 595 resulted in potent growth 
inhibition and significant tumor mitotic arrest. The results of this study encouraging further researches to evaluate AMG 595 in patients with EGFRvIII-expressing glioblastoma [75]. NCT01475006 is an open label non-randomized Phase I first-in-human study evaluating safety, tolerability, pharmacokinetics and pharmacodynamics of AMG 595 in patients with recurrent malignant glioma expressing mutant (EGFRvIII).

MR1-1 is a novel recombinant immunotoxin that targets EGFRvIII positive GBM tumor-specific antigen [77]. NCT01009866 is open label lhase I study of immunotoxin, MR1-1 to determine maximum tolerated dose and dose limiting toxicity of MR1-1KDEL when delivered intracerebrally by convection-enhanced delivery (CED) in patients with supratentorial malignant brain tumor.

\section{Vascular Endothelial Growth Factor Receptor (VEGFR) Inhibitors}

VEGF is the most potent growth factor mediating tumor angiogenesis [81]. When VEGF binds to the VEGFR, activation cascade of PLC-PKC-Raf kinase, MEK-MAPK and PI3K-Akt pathways will be resulted, this cellular cascade enhances endothelial cell survival, differentiation, migration with subsequent formation of tumor microvasculature. In glioblastoma, VEGF levels are shown to be high and overexpression of VEGFR has been associated with poor prognosis. It is also shown that levels of VEGF tend to be higher in patients with recurrent glioblastomas, So VGEF believed to be promising target in the treatment these patients [82-84]. The pathways of VEGF can be targeted directly or by inhibiting its receptor. So the two large group targeting VEGF and /or its receptor are monoclonal antibodies and TKIs.

\section{1- Monoclonal Antibodies Targeting VEGF}

Bevacizumab is a recombinant humanized monoclonal antibody that binds to VEGF with high affinity and specificity, preventing interaction with its receptors, resulting in suppression of VEGF signaling [85]. In GBM bevacizumab has been extensively studied especially for progressed and recurrent cases after front line standard definitive therapy. In May 2009 bevacizumab received US Food and Drug Administration (FDA) approval for use as a single agent in patients with glioblastoma who have progressive disease after initial standard treatment based on results of clinical trials support its use. Table 3 below summarizes different studies of bevacizumab in glioblastoma.

Table 3. Studies of bevacizumab in glioblastoma

\begin{tabular}{|c|c|c|}
\hline Author/year & Type of study & Study title \\
\hline Kreisl et al (2009) [86] & PhaseII & $\begin{array}{l}\text { Single-agent bevacizumab followed by bevacizumab plus irinotecan } \\
\text { at tumor progression in recurrent glioblastoma. }\end{array}$ \\
\hline Friedman et al (2009) [87] & PhaseII & Bevacizumab alone and in combination with irinotecan in recurrent glioblastoma \\
\hline Gilbert et al (2014) [88] & PhaseIII & A randomized trial of bevacizumab for newly diagnosed glioblastoma \\
\hline Chinot et al (2014) [98] & PhaseIII & Bevacizumab plus radiotherapy-temozolomide for newly diagnosed glioblastoma \\
\hline Taal et al (2014) [90] & PhaseII & $\begin{array}{c}\text { Single-agent bevacizumab or lomustine versus a combination of bevacizumab plus } \\
\text { lomustine in patients with recurrent glioblastoma (BELOB trial) }\end{array}$ \\
\hline EORTC 26101(ongoing) & PhaseIII & $\begin{array}{l}\text { Combination of Bevacizumab and Lomustine in Patients With First } \\
\text { Recurrence of a Glioblastoma (NCT01290939 protocol) }\end{array}$ \\
\hline
\end{tabular}


Two phase II studies were conducted to evaluate the efficacy of bevacizumab in progressive or recurrent GBM after front line standard therapy. First one conducted on 48 patients with recurrent glioblastoma to evaluate efficacy of single agent bevacizumab. The results were accepted by the author who concluded that single-agent bevacizumab has significant biologic and anti-glioma activity in patients with recurrent GBM [86]. The second study were undertaken by Friedman and colleagues to evaluate the efficacy of bevacizumab alone or in combination with irinotecan in patients with recurrent GBM where 167 patients with recurrent glioblastoma were randomly assigned to receive bevacizumab alone or in combination with irinotecan once every 2 weeks, the findings of this study showed that bevacizumab alone or in combination with irinotecan was well tolerated and effective in patients with recurrent glioblastoma [87]. Based on the previous two studies, US FDA approved bevacizumab as a single agent in patients with recurrent glioblastoma. Following these phase II studies, bevacizumab was evaluated in phase III trial for patients with newly diagnosed GBM. Gilbert and colleagues conducted phase III study on patients with newly diagnosed glioblastoma with addition of bevacizumab (treatment arm) or placebo( control arm) to standard concurrent chemo radiotherapy but there was no improvement in overall survival compared to the standard treatment arm, also prolonged progression-free survival did not reach the pre specified improvement target [88]. Another phase III study by Chinot and colleagues with nearly the same protocol of Gilbert study, the results nearly the same with addition that, the rate of adverse events was higher with bevacizumab arm [89]. The final finding from these two studies is no benefit from addition of bevacizumab to standard concurrent chemo-radiotherapy for patients with newly diagnosed glioblastoma. NCT01290939 protocol is ongoing phase III trial to explore the combination of bevacizumab and lomustine in patients with first recurrence of GBM. This trial is ongoing but not recruiting participants.

\section{2- Receptor Tyrosine Kinase Inhibitors (TKIs) Targeting VEGFR in Glioblastoma}

Receptor (TKIs) is small-molecules blocking the tyrosine kinase activation site of VEGFR. In recurrent high-grade glioma different receptor TKIs have been investigated e.g Sunitinib, pazopanib, cediranib, vandetanib and sorafenib which are multi-kinase inhibitors of VEGFR that have been evaluated in glioblastoma. These agents are inhibitors of other relevant receptors as EGFR, PDGFR, KIT, RET and RAF oncogens [97]. These VEGFR TKIs have been evaluated in number of phase I/II studies to determine its role in glioblastoma, below different TKIs targeting VEGFR will be summarized.

Sunitinib is a tyrosine kinase inhibitor with direct anti-tumor and anti-angiogenesis activity targeting VEGFR $(1,2)$, PDGFR $(\alpha, \beta)$, c-kit, bFGF, FLT3 and RET proto oncogens [91]. In glioblastoma phase II studies of sunitinib showed limited or insufficient activity this drug in recurrent GBM $[92,93]$.

Pazopanib is a second generation tyrosine kinase inhibitor that targets VEGFR $(1,2,3)$, PDGFR $(\alpha, \beta)$, and c-Kit [99]. Phase II single arm study was conducted to evaluate efficacy and safety of pazopanib for patients with recurrent GBM not exposed to previous antiangiogenic therapy, the primary end point was progression free survival (efficacy) and secondary end point was tolerability. Although treatment was tolerated the author concluded that pazopanib did not prolong progression free survival [94].

Table 4. Receptor tyrosin kinas inhibitors targeting VEGFR in glioblastoma

\begin{tabular}{|c|c|c|}
\hline Agent & Author/year & Study type \\
\hline Sunitinib & Neynsetal(2011)[92] & PhaseII \\
& Preisletal(2013)[93] & PhaseII \\
Pazopanib & Pwamotoetal(2010)[94] & PhaseII \\
& & PhaseIII \\
Cediranib & Batcheloretal(2010)[95] & PhaseII \\
& Batcheloretal(2013)[96] & PhaseI \\
Vandetanib & Chedaetal(2015)[97] & PhaseII \\
& PhaseII \\
\hline
\end{tabular}


Cediranib is a pan-VEGFR TKIs; also it has additional activity against PDGF $(\beta)$ and c-Kit. Cediranib has the ability to pass the $\mathrm{BBB}$, but due active efflux via $\mathrm{BBB}$ transporters there are limited cerebrospinal fluid concentrations [100, 101]. In glioblastoma, cediranib was evaluated in both phase II and phase III studies. The first phase II trial was conducted by Batchelor and colleagues to evaluate the efficacy of cediranib monotherapy for patients with recurrent glioblastoma, the primary end point was 6 month progression free survival, the toxicities were manageable and the results were encouraging and the author recommend further investigations as cediranib was effective against recurrent GBM [95].The results obtained in previous study encouraged the same author to undergo a phase III placebo-controlled, partially blinded clinical trial to determine the efficacy of cediranib as monotherapy or in combination with lomustine versus lomustine in patients with recurrent glioblastoma. Although the results of phase II trial was encouraging the results of phase III not and the author concluded that the primary end point of PFS prolongation with cediranib either as monotherapy or in combination with lomustine versus lomustine not achieved in patients with recurrent glioblastoma, but he noticed that cediranib showed evidence of clinical activity on some secondary end points as time to deterioration in neurologic status and corticosteroid-sparing effects [96].

Sorafenib is a multi-kinase inhibitor which has demonstrated antiangiogenic and antiproliferative activity in a number of invitro and invivo models. Also sorafenib has good BBB permeability $[102,103]$. Invitro studies showed that there was evidence of a selective action of sorafenib on glioblastoma cells even if the results from first phase II clinical trials did not demonstrate this efficacy [105]. Initially phase I study was conducted to determine the maximum tolerated dose of sorafenib in patients with recurrent malignant glioma. The MTD of sorafenib given orally BID on a continuous basis was established as $600 \mathrm{mg}$ in patients did not receive EIAEDs and $800 \mathrm{mg}$ in patients did [103]. Phase II studies of sorafinib in GBM was conducted to evaluate its efficacy if combined with other agents. Combination of sorafenib and temozolomide as salvage therapy for recurrent glioblastoma revealed no benefit from addition of sorafenib to temozolomide $[106,107,108]$. Also sorafinib when combined with erlotinib in phase II trial conducted by Peereboom et al, there were significant pharmacokinetic interactions resulting in negatively impact on efficacy of the combination regimen [109].

\section{3- Other VEGF Inhibitors}

There are other agents with anti VEGF activity believed to have a role in treatment of GBM. Afliberept, a recombinant fusion protein although has a several hundred-fold greater affinity for VEGF than bevacizumab, phase II study to assess its efficacy for patients with recurrent glioblastoma after standard front line therapy showed non accepted results and the conclusion was that aflibercept as a single agent has minimal activity in recurrent glioblastoma [110,111]. Thalidomide, an anti-angiogenesis primary targeting bFGF and VEGF had been evaluated early in the treatment of gliomas but the results were unimpressive and most clinical studies demonstrated no efficacy $[112,113]$. Cilengitide is a selective inhibitor of $\alpha \mathrm{v} \beta 3$ and $\alpha \mathrm{v} \beta 5$ integrins. Although phase I and II clinical studies of cilengitide showed that the drug was well-tolerated and had evidence of antitumor activity in patients with newly diagnosed and recurrent glioblastoma [114,115]. Large phase III study was conducted by Stupp et al, where a total of 3471 patients were enrolled. The final interpretation was that, the addition of cilengitide to temozolomide chemoradiotherapy did not improve outcomes and the author recommended that cilengitide should not be further developed as an anticancer drug [116].

\subsection{Inhibitors of Ras Pathway}

Ras pathway and its associated proteins are important regulators of normal cell growth and malignant cell transformation. Overexpression or upregulation of Ras contributes to oncogenesis in a variety of cancers. In GBM Ras mutations are rare, but inhibition of Ras can still be effective because of its action as a second messenger in signaling cascades of different growth factors as EGFR, PDGFR, and VEGFR and so it is involved in the pathogenesis of malignant gliomas [117, 118]. Farnesyltransferase inhibitors (FTIs) are posttranscriptional modifiers of Ras. Invitro studies of FTIs in glioblastoma have demonstrated significantly decrease cellular proliferation, cell cycle arrest, and even stimulate apoptosis [119]. Tipifarnib and Lonafarnib are selective FTIs, phase I/ II studies of both drugs in glioblastoma showed disappointing results compared to prior studies of temozolomide monotherapy for recurrent glioblastoma, warranting further researches, in addition, the findings showed that the effect of lonafarnib appeared to be greater than tipifarnib [120, 121].

\subsection{Inhibitors of Mammalian Target of Rabamycin (mTOR)}

PTEN downregulate activity of PI3K/ATK pathway downstream signaling of mTOR which control cell growth and cell cycle progression, PTEN mutation and deletion lead to unregulated PI3K/ATK with subsequently increased mTOR activity, PTEN tumor suppressor mutations and deletions present in $\sim 30-40 \%$ of GBM cases $[118,122,123]$. Everolimus, Sirolimus and temsirolimus are inhibitors of mTOR with different mechanisms of action, data from phase II studies showed limited activity of these agents either as monotherapy or combined with other agents in newly diagnosed or recurrent glioblastoma patients [45, 124, 125]. 


\section{Other Molecular Targets Involved in Treatment of GBM}

Tamoxifen is a highly lipid soluble nonsteroidal agent primary used in treatment of estrogen receptor-positive breast cancer, so it can readily crosses the BBB. Beside inhibition of estrogen receptor it also inhibits other pathways, including protein kinas C [126, 127]. Early in 1990s phase II clinical trials were conducted to test efficacy of tamoxifen in patients with recurrent glioblastoma. Couldwell and colleagues used high dose tamoxifen in recurrent GBM, there were high rate of radiologic response, complete response represented $20 \%$ and partial response was 25 but overall survival rate was 6,8 months and progression free time was 3.3 months [128]. Another phase II study was conducted by Brandes et al to test high-dose tamoxifen with procarbazine in 28 patients with recurrent glioblastoma, the results were nearly the same to that obtained in Couldwell study [129]. The findings of study undertaken by Di Cristofori and colleagues who test tamoxifen in combination with dense dose temozolomide in patient with recurrent glioblastoma were promising [130] but further researches should consider the role of tamoxifen in glioblastoma more vigorously in the future.

Enzastaurin, a small inhibitor of protein kinas $\mathrm{C}$ with high BBB permeability due to its lipid solubility has been used for the treatment of a variety of tumors. In an early phase clinical study, promising results as regard to radiographic response rates seen in patients with recurrent high-grade gliomas treated with enzastaurin alone directed Wick and colleagues to conduct a phase III clinical study of enzastaurin compared to lomustine in 266 patients with recurrent glioblastoma, but the study was terminated early secondary to the lack of enzastaurin efficacy compared with lomustine $[52,131]$.

\section{Gene Therapy}

The process of Gene therapy can be done by introduction of a therapeutic gene or manipulation of disease-related gene such as abscission of an activated oncogene within target cells. Glioblastoma is an ideal target for gene therapy as the current standard therapies have minimal effects in its treatment [132]. A review article have been explored to discusses various gene therapies for patients with glioblastoma focusing on the strategies used for gene delivery as well as novel therapeutic approaches, an overview of these strategies was presented with discussion of gene therapy-based strategies to attack such a complex genetic targets as glioblastoma [133]. Although gene therapy showed significant anti-cancer effects in other types of cancers, its efficacy in glioblastoma needs further researches to support therapeutic standard therapy [132].

\section{Summary}

Glioblastoma remains one of the most frequent and most clinically challenging primary brain tumors. The current standard treatment after maximal safe surgical resection is concurrent chemoradiotherapy with temozolomide and radiation therapy. In the last few decades, advancements in understanding of the pathogenesis of molecular pathways in GBM have led to new era of exciting possibilities to improve treatment outcome. In this review, recent developments around the molecular targeted therapy of glioblastoma were summarized. Currently available molecularly targeted therapies for GBM have had poor to modest activity in clinical trials, but with continuous development of newer agents, combination regimens are the next step in improving outcomes of this fatal disease. Also stratifying patients based on molecular profiles of their tumor will allow a tailored treatment approach when using molecular targeted therapy. Furthermore, identifying molecular biomarkers of treatment response will allow this novel therapy to fulfill their therapeutic promise.

\section{REFERENCES}

[1] Ostrom Q T, Gittleman H, Liao P, et al: CBTRUS Statistical Report: Primary Brain and Central Nervous System Tumors Diagnosed in the United States in 2007-2011. Neuro-Oncology 16:iv1-iv63, 2014

[2] Omuro A, DeAngelis LM: Glioblastoma and other malignant gliomas: a clinical review. JAMA 310:1842-50, 2013

[3] Lacroix M, Abi-Said D, Fourney DR, et al: A multivariate analysis of 416 patients with glioblastoma multiforme: prognosis, extent of resection, and survival. J Neurosurg 95:190-198, 2001

[4] Theeler BJ, Yung WK, Fuller GN, et al: Moving toward molecular classification of diffuse gliomas in adults. Neurology 79:1917-26, 2012

[5] Ohgaki H, Kleihues P: Population-based studies on incidence, survival rates, and genetic alterations in astrocytic and oligodendroglial gliomas. J Neuropathol Exp Neurol 64: 479-489, 2005

[6] Bredel M, Scholtens DM, Harsh GR, et al: A network model of a cooperative genetic landscape in brain tumors. JAMA 302:261-75, 2009

[7] Phillips HS, Kharbanda S, Chen R, et al: Molecular subclasses of high-grade glioma predict prognosis, delineate a pattern of disease progression, and resemble stages in neurogenesis. Cancer cell 9:157-173, 2006

[8] Verhaak RG, Hoadley KA, Purdom E, et al: Integrated genomic analysis identifies clinically relevant subtypes of glioblastoma characterized by abnormalities in PDGFRA, IDH1, EGFR, and NF1. Cancer cell 17:98-110, 2010

[9] Zheng S, Chheda MG, Verhaak RG: Studying a complex tumor: potential and pitfalls. Cancer J 18:107-114, 2012

[10] Paugh BS, Qu C, Jones C, et al: Integrated molecular genetics profiling of pediatric high-grade gliomas reveals key differences with the adult disease. J Clin Oncol 28: 
3061-3068, 2010

[11] Singh D, Chan JM, Zoppoli P, et al: Transforming fusions of FGFR and TACC genes in human glioblastoma. Science 337:1231-1235, 2012

[12] Ohgaki H, Kleihues P: The definition of primary and secondary glioblastoma. Clin Cancer Res 19:764-772, 2013

[13] Wong AJ, Bigner SH, Bigner DD, et al: Increased expression of the epidermal growth factor receptor gene in malignant gliomas is invariably associated with gene amplification. Proc Natl Acad Sci USA 84:6899-6903, 1987

[14] Yan H, Parsons DW, Jin G, et al: IDH1 and IDH2 mutations in gliomas. N Engl J Med 360:765-773, 2009

[15] Vivanco I, Robins HI, Rohle D, et al: Differential sensitivity of glioma versus lung cancer-specific EGFR mutations to EGFR kinase inhibitors. Cancer Discov 2: 458-471, 2012

[16] Lee JC, Vivanco I, Beroukhim R, et al: Epidermal growth factor receptor activation in glioblastoma through novel missense mutations in the extracellular domain. PLoS Med 3: 485,2006

[17] Sugawa N, Ekstrand AJ, James CD, et al: Identical splicing of aberrantepidermal growth factor receptor transcripts from amplified rearranged genes in human glioblastomas. Proc Natl Acad Sci USA 87:8602-8606, 1990

[18] Nishikawa R, Ji XD, Harmon RC, et al: A mutant epidermal growth factor receptor common in human glioma confers enhanced tumorigenicity. Proc Natl Acad Sci USA 91:7727-7731, 1994

[19] Yarden Y, Pines G: The ERBB network: at last, cancer therapy meets systems biology. Nat Rev Cancer 12: 553-563, 2012

[20] Lv S, Teugels E, Sadones J, et al: Correlation of EGFR, IDH1 and PTEN status with the outcome of patients with recurrent glioblastoma treated in a phase II clinical trial with the EGFR-blocking monoclonal antibody cetuximab. Int J Oncol 41:1029-1035, 2012

[21] Ramos TC, Figueredo J, Catala M, et al: Treatment of high-grade glioma patients with the humanized anti-epidermal growth factor receptor (EGFR) antibody h-R3: report from a phase I/II trial. Cancer Biol Ther 5: 375-379, 2006

[22] Hasselbalch B, Lassen U, Hansen S, et al: Cetuximab, bevacizumab, and irinotecan for patients with primary glioblastoma and progression after radiation therapy and temozolomide: a phase II trial. Neuro Oncol 12:508-516, 2010

[23] Wersall P, Ohlsson I, Biberfeld P, et al: Intratumoral infusion of the monoclonal antibody, $\mathrm{mAb} 425$, against the epidermal-growth factor receptor in patients with advanced malignant glioma. Cancer Immunol Immunother. 44:157-164, 1997

[24] Reardon DA, Wen PY and Mellinghoff I K: Targeted molecular therapies against epidermal growth factor re ceptor: Past experiences and challenges Neuro-Oncology 16:viii7-viii13, 2014

[25] Kreisl TN, McNeill KA, Sul J, et al: A phase I/II trial of vandetanib for patients with recurrent malignant glioma. Neuro Oncol 14: 1519-1526, 2012
[26] Reardon DA, Conrad CA, Cloughesy T, et al: Phase I study of AEE788, a novel multitarget inhibitor of ErbB- and VEGF-receptor-family tyrosine kinases, in recurrent glioblastoma patients. Cancer Chemother Pharmacol 69:1507-1518, 2012

[27] Bode U, Massimino M, Bach F, et al: Nimotuzumab treatment of malignant gliomas. Expert Opin Biol Ther $12: 1649-59,2012$

[28] Patel D, Lahiji A, Patel S, et al: Monoclonal antibody cetuximab binds to and down-regulate sconstitutively activated epidermal growth factor receptor vIII on the cell surface. Anticancer Res 27:3355-66, 2007

[29] Padfield E, Ellis H P and. Kurian K M.: Current therapeutic advances targeting EGFR and EGFRvIII in glioblastoma.fronier in oncology 2015

[30] Halatsch ME,Gehrke EE,Vougioukas VI, et al: Inverse correlation of epidermal growth factor receptor messenger RNA induction and suppression of anchorage-independent growth by OSI-774, an epidermal growth factor receptor tyrosine kinase inhibitor, in glioblastoma multiforme cell lines. J Neurosurg 100:523-33, 2004

[31] Quatrale AE, Porcell iL and Silvestris N: EGFR tyrosine kinase inhibitors in cancer treatment: invitro and in vivo evidence. Front Biosci 16:1962-72, 2011

[32] Gan HK, KayeAH, Luwor RB:The EGFRv III variant in glioblastoma multi- forme. J ClinNeurosci 16:748-54, 2009

[33] Rich JN, Rasheed BK, Yan H: EGFR mutations and sensitivity to gefitinib.N Engl J Med 351:1260-1, 2004

[34] Rich JN, Reardon DA, Peery T, et al: Phase II trial of gefitinib in recurrent glioblastoma. J Clin Oncol 22:133-142, 2004

[35] Reardon DA, Quinn JA, Vredenburgh JJ, et al: Phase 1 trial of gefitinib plus sirolimus in adults with recurrent malignant glioma. Clin Cancer Res 12: 860-868, 2006

[36] Prados MD, Yung WK, Wen PY, et al: Phase-1 trial of gefitinib and temozolomide in patients with malignant glioma: a North American brain tumor consortium study. Cancer Chemother Pharmacol 61:1059-1067, 2008

[37] Hegi ME, Diserens AC, Bady P, et al: Pathway analysis of glioblastoma tissue after preoperative treatment with the EGFR tyrosine kinase inhibitor gefitinib--a phase II trial. Mol Cancer Ther. 10: 1102-1112, 2011

[38] Uhm JH, Ballman KV, Wu W, et al: Phase II Evaluation of Gefitinib in Patients With Newly Diagnosed Grade 4 Astrocytoma: Mayo/North Central Cancer Treatment Group Study N0074. Int J Radiat Oncol Biol Phys 80:347-353, 2011

[39] Chakravarti A, Wang M, Robins HI, et al: a phase $1 / 2$ study of radiation therapy with concurrent gefitinib for newly diagnosed glioblastoma patients. Int J Radiat Oncol Biol Phys 85:1206-1211, 2013

[40] Prados MD, Lamborn KR, Chang S, et al: Phase 1 study of erlotinib $\mathrm{HCl}$ alone and combined with temozolomide in patients with stable or recurrent malignant glioma. Neuro Oncol 8:67-78, 2006

[41] Krishnan S, Brown PD, Ballman KV, et al: Phase I trial of erlotinib with radiation therapy in patients with glioblastoma multiforme: results of North Central Cancer Treatment Group protocol N0177. Int J Radiat Oncol Biol Phys 65:1192-1199, 2006 
[42] Brown PD, Krishnan S, Sarkaria JN, et al: Phase I/II trial of erlotinib and temozolomide with radiation therapy in the treatment of newly diagnosed glioblastoma multiforme: North Central Cancer Treatment Group Study N0177. J Clin Oncol 26:5603-5609, 2008

[43] Prados MD, Chang SM, Butowski N, et al: Phase II study of erlotinib plus temozolomide during and after radiation therapy in patients with newly diagnosed glioblastoma multiforme or gliosarcoma. J Clin Oncol 27:579-584, 2009

[44] Peereboom DM, Shepard DR, Ahluwalia MS, et al: Phase II trial of erlotinib with temozolomide and radiation in patients with newly diagnosed glioblastoma multiforme. J Neurooncol 98: 93-99. 2010, 2010

[45] Reardon DA, Desjardins A, Vredenburgh JJ, et al: Phase 2 trial of erlotinib plus sirolimus in adults with recurrent glioblastoma J Neurooncol 96:219-230, 2010

[46] Raizer JJ, Abrey LE, Lassman AB, et al: A phase II trial of erlotinib in patients with recurrent malignant gliomas and nonprogressive glioblastoma multiforme postradiation therapy. Neuro Oncol 12:95-103, 2010

[47] van den Bent MJ, Brandes AA, Rampling R, et al: Randomized phase II trial of erlotinib versus temozolomide or carmustine in recurrent glioblastoma: EORTC brain tumor group study 26034. J Clin Oncol 27:1268-1274, 2009

[48] Yung WK, Vredenburgh JJ, Cloughesy TF, et al: Safety and efficacy of erlotinib in first-relapse glioblastoma: a phase II open-label study.Neuro Oncol 12:1061-1070, 2010

[49] Wen PY, Chang SM, Lamborn KR, et al: Phase I/II study of erlotinib and temsirolimus for patients with recurrent malignant gliomas:North American Brain Tumor Consortium trial 04-02. Neuro Oncol 16:567-578, 2014

[50] Eisenstat D. D, Nabors L. B, Mason W. P et al: A phase II study of daily afatinib (BIBW 2992) with or without temozolomide (21/28 days) in the treatment of patients with recurrent glioblastoma. J Clin Oncol 29: 2011 (suppl; abstr 2010)

[51] Reardon DA, Nabors LB, Mason WP, et al: Phase I/Randomized phase II study of afatinib, an irreversible ErbB family blocker, with or without protracted temozolomide in adults with recurrent glioblastoma. Neuro Oncol 0:1-10, 2014

[52] Lau D, Magill S T and Aghi M K. Molecularly targeted therapies for recurrent glioblastoma:current and future targets. Neurosurg Focus 37 (6): E15, 2014

[53] Chen Y, Wang M, Zhong W, Zhao J: Pharmacokinetic and pharmacodynamic study of Gefitinib in a mouse model of non-small-cell lung carcinoma with brain metastasis. Lung Cancer 82:313-318, 2013

[54] Paul B, Trovato J A and Thompson J: Lapatinib: A dual tyrosine kinase inhibitor for metastatic breast cancer. American Journal of Health-System Pharmacy 18:1703-1710, 2008

[55] Thiessen B, Stewart C, Tsao $M$ et al: A phase I/II trial of GW572016 (lapatinib) in recurrent glioblastoma multiforme: clinical outcomes, pharmacokinetics and molecular correlation. Cancer Chemotherapy and Pharmacology. 65(2):353-61, 2010

[56] Reardon D A, Groves M D, Wen P Y.: A Phase I/II Trial of
Pazopanib in Combination with Lapatinib in Adult Patients with Relapsed Malignant Glioma. Clin Cancer Res 19(4); 900-8, 2012

[57] Zahonero C, Aguilera P, Ramírez-Castillejo C: Preclinical Test of Dacomitinib, an Irreversible EGFR Inhibitor, Confirms Its Effectiveness for Glioblastoma. Mol Cancer Ther. 14(7):1548-58, 2015

[58] López-Tarruell S, Jerez Y, Márquez-Rodas I and Martín M: Neratinib (HKI-272) in the treatment of breast cancer. Future Oncol 8(6), 671-681, 2012

[59] Sequist LV, Besse B, Lynch T J: Neratinib, an Irreversible Pan-ErbB Receptor Tyrosine Kinase Inhibitor: Results of a Phase II Trial in Patients With Advanced Non-Small-Cell Lung Cancer .jour clin oncol 28. 18, 2010

[60] Martinelli E, De Palma R, Orditura M et al. Anti-epidermal growth factor receptor monoclonal antibodies in cancer therapy. Clin Exp Immunol 158(1): 1-9, 2009

[61] Taylor T E, Furnari F B, Cavenee W K: Targeting EGFR for Treatment of Glioblastoma: Molecular Basis to Overcome Resistance. Curr Cancer Drug Targets 12(3): 197-209.2012

[62] Combs S E, Heeger S, Haselmann R et al: Treatment of primary glioblastoma multiforme with cetuximab, radiotherapy and temozolomide (GERT) - phase I/II trial: study protocol. BMC Cancer 6:133, 2006

[63] Neyns B, Sadones J, Joosens E, et al: Stratified phase II trial of cetuximab in patients with recurrent thigh-grade glioma. Ann Oncol 20:1596-03, 2009

[64] Hasselbalch B, Lassen U, Hansen S, et al: Cetuximab, bevacizumab, and irinotecan for patients with primary glioblastoma and progression after radiation therapy and temozolomide: a phase II trial. Neuro Oncol 12:508-516, 2010

[65] Lv S, Teugels E, Sadones J, et al: Correlation of EGFR, IDH1 and PTEN status with the outcome of patients with recurrent glioblastoma treated in a phase II clinical trial with the EGFR-blocking monoclonal antibody cetuximab. Int J Oncol 41:1029-1035, 2012

[66] Mortensen J H, Maria J, Pilgaard L et al: Targeted Antiepidermal Growth Factor Receptor (Cetuximab) Immunoliposomes Enhance Cellular Uptake In Vitro and Exhibit Increased Accumulation in an Intracranial Model of Glioblastoma Multiforme. Jour Dru Deliv 2013

[67] Diaz A, Blanco R, Lemm M et al: Preclinical Efficacy of Nimotuzumab, an Anti-EGFR Monoclonal Antibody as a Single Agent Therapy in Human GBM U87MG Xenografts. Journal of Cancer Therapy 3: 245-255, 2012

[68] Hong J, Peng Y, Liao Y et al: Nimotuzumab prolongs survival in patients with malignant gliomas: A phase I/II clinical study of concomitant radiochemotherapy with or without nimotuzumab. Exper therap med 4: 151-157, 2012

[69] Solomón M T, Selva J C, Figueredo J et al: Radiotherapy plus nimotuzumab or placebo in the treatment of high grade glioma patients: results from a randomized, double blind trial. MC Cancer 13:299, 2013

[70] Wang Y, Pan L, Sheng X f et al. Nimotuzumab, a humanized monoclonal antibody specific for the EGFR, in combination with temozolomide and radiation therapy for newly 
diagnosed glioblastoma multiforme: First results in Chinese patients. Asia-Pacific Journal of Clinical Oncology 2014

[71] Solomon M T, Miranda N, Jorrín E et al: Nimotuzumab in combination with radiotherapy in high grade glioma patients. Cancer Biology \& Therapy 15:5, 504-509, 2014

[72] Westphal M, Bach F: Final results of a randomized phase III trial of nimotuzumab for the treatment of newly diagnosed glioblastoma in addition to standard radiation and chemotherapy with temozolomide versus standard radiation and temoziolamide. ASCO Annual Meeting. J Clin Oncol. 30(Suppl):Abstr 2033, 2012

[73] Li L, Quang TS, Gracely EJ, et al: A Phase II study of anti-epidermal growth factor receptor radioimmunotherapy in the treatment of glioblastoma multiforme. J Neurosurg. 113(2):192-8, 2010

[74] Johns T G, Mcay M J, Cvrljevic A N, et al: MAb 806 Enhances the Efficacy of Ionizing NRadiation in Glioma Xenografts Expressing the de2-7 Epidermal growth Factor Receptor. -Int. J. Radiation Oncology Biol. Phys 78( 2) $572-578,2010$

[75] Hamblett KJ, Kozlosky CJ, Siu S et al: AMG 595, an Anti-EGFRvIII Antibody-Drug Conjugate, Induces Potent Antitumor Activity against EGFRvIII-Expressing Glioblastoma. Mol Cancer Ther. 14(7):1614-24, 2015

[76] Ding D, Kanaly C W, Bigner D, et al: Convection-enhanced delivery of free adolinium with the Recombinant immunotoxin MR1-1. J Neurooncol. 98(1): 1-7, 2010

[77] Choi B D, Kuanb C T, Cai M et al: Systemic administration of a bispecific antibody targeting EGFRvIII successfully treats intracerebral glioma. PNAS 110(1)270-275, 2013

[78] Iniesta C B, Carpeño J, Saenz E C, et al: Long Term Responses with Cetuximab Therapy in Glioblastoma Multiforme. Cancer Biology \& Therapy 5:8, 912-914, 2006

[79] Patel D, Lahiji A, Patel S, et al: Monoclonal antibody cetuximab binds to and down-regulates constitutively activated epidermal growth factor receptor vIII on the cell surface. Anticancer Res 27: 3355-3366, 2007

[80] Ramakrishnan M S, Eswaraiah A, Crombet $\mathrm{T}$ et al: Nimotuzumab, a promising therapeutic monoclonal for treatment of tumors of epithelial origin. MAbs 1(1): 41-48, 2009

[81] Folkins C, Man S, Xu P, et al: Anticancer therapies combining antiangiogenic and tumor cell cytotoxic effects reduce the tumor stem-like cell fraction in glioma xenograft tumors. Cancer Res 67:3560-3564, 2007

[82] Kerbel RS: Tumor angiogenesis. N Engl J Med 358:2039-2049, 2008

[83] Nam DH, Park K, Suh YL and Kim JH: Expression of VEGF and brain specific angiogenesis inhibitor-1 in glioblastoma: prognostic significance. Oncol Rep 11:863-869, 2004

[84] Salmaggi A, Eoli M, Frigerio S, et al: Intracavitary VEGF, bFGF, IL-8, IL-12 levels in primary and recurrent malignant glioma. J Neurooncol 62: 297-303, 2003

[85] Ferrara N, Hillan KJ, Gerber HP and Novotny W: Discovery and development of bevacizumab, an anti-VEGF antibody for treating cancer. Nat Rev Drug Discov 3:391-400, 2004

[86] Kreisl TN, Kim L, Moore K, et al: Phase II trial of single-agent bevacizumab followed by bevacizumab plus irinotecan at tumor progression in recurrent glioblastoma. J Clin Oncol 27: 740-5, 2009

[87] Friedman HS, Prados MD, Wen PY, et al: Bevacizumab alone and in combination with irinotecan in recurrent glioblastoma. J Clin Oncol 27: 4733-40, 2009

[88] Gilbert MR, Dignam JJ, Armstrong TS, et al: A randomized trial of bevacizumab for newly diagnosed glioblastoma. $\mathrm{N}$ Engl J Med 370:699-708, 2014

[89] Chinot OL, Wick W, Mason W, et al: Bevacizumab plus radiotherapy-temozolomide for newly diagnosed glioblastoma. N Engl J Med 370:709-22, 2014

[90] Taal W, Oosterkamp HM, Walenkamp AM, et al: Single-agent bevacizumab or lomustine versus a combination of bevacizumab plus lomustine in patients with recurrent glioblastoma (BELOB trial): a randomised controlled phase 2 trial. Lancet Oncol 15:943-53, 2014

[91] Chow LQ, Eckhardt SG: Sunitinib: from rational design to clinical efficacy. J Clin Oncol 25:884-96, 2007

[92] Neyns B, Sadones J, Chaskis C, et al: study of Phase II sunitinib malate in patients with recurrent high-grade glioma. J Neurooncol 103:491-501, 2011

[93] Kreisl TN, Smith P, Sul J, et al: Continuous daily sunitinib for recurrent glioblastoma. J Neuro oncol 111(1):41-8, 2013

[94] Iwamoto FM, Lamborn KR, Robins HI, et al: Phase II trial of pazopanib (GW786034), an oral multi-targeted angiogenesis inhibitor, for adults with recurrent glioblastoma (North American Brain Tumor Consortium Study 06-02). Neuro Oncol 12:855-61, 2010

[95] Batchelor TT, Mulholland P, Neyns B, et al: Phase III randomized trial comparing the efficacy of cediranib as monotherapy, and in combination with lomustine, versus lomustine alone in patients with recurrent glioblastoma. J Clin Oncol 31: 3212-8, 2013

[96] Cheda MG, Wen PY, Hochberg FH, et al: Vandetanib plus sirolimus in adults with recurrent glioblastoma: results of a phase I and dose expansion cohort study. J Neurooncol 121:627-34, 2015

[97] Weather S and De Groot J: VEGF Manipulation in Glioblastoma. Cancer Network. 2015

[98] Bhat KP, Balasubramaniyan V, Vaillant B, et al: Mesenchymal differentiation mediated by NF-kappaB promotes radiation resistance in glioblastoma. Cancer Cell 24:331-46, 2013

[99] Sloan B, Scheinfeld NS: Pazopanib, a VEGF receptor tyrosine kinase inhibitor for cancer therapy. Curr Opin Investig Drugs 9(12):1324-1335, 2008

[100] Wang T, Agarwal S, Elmquist WF: Brain distribution of cediranib is limited by active efflux at the blood-brain barrier. J Pharmacol Exp Ther 341: 386-395, 2012

[101] Wedge SR, Kendrew J, Hennequin LF, et al: AZD2171: a highly potent, orally bioavailable, vascular endothelial growth factor receptor- 2 tyrosine kinase inhibitor for the treatment of cancer. Cancer Res 65: 4389-4400, 2005

[102] Kast RE, Boockvar JA, Brüning A, et al: A conceptually new 
treatment approach for relapsed glioblastoma: coordinated undermining of survival paths with nine repurposed drugs (CUSP9) by the International Initiative for Accelerated Improvement of Glioblastoma Care. Oncotarget 4: 502-530, 2013

[103] Nabors L. B, Supko J. G, Rosenfeld M, et al: Phase I trial of sorafenib in patients with recurrent or progressive malignant glioma. Neuro-Oncology 2011

[104] Galanis E, Anderson S. K, Lafky J M, et al: Phase II Study of Bevacizumab in Combination with Sorafenib in Recurrent Glioblastoma (N0776): A North Central Cancer Treatment Group Trial. Clin Cancer Res 19(17), 2013

[105] Carra E, Barbieri F, Marubbi D, et al: Sorafenib selectively depletes human glioblastoma tumor-initiating cells from primary cultures. Cell Cycle 12:3, 491-500, 2013

[106] Reardon DA, Vredenburgh JJ, Desjardins A, et al: Effect of CYP3A-inducing antiepileptics on sorafenib exposure: results of a phase II study of sorafenib plus daily temozolomide in adults with recurrent glioblastoma. J Neurooncol 101:57-66, 2011

[107] Van den Bent MJ, Taal W: Are we done with dose-intense temozolomide in recurrent glioblastoma? Neuro Oncol 16: 1161-1163, 2014

[108] Zustovich F, Landi L, Lombardi G, et al: Sorafenib plus daily low-dose temozolomide for relapsed glioblastoma: a phase II study. Anticancer Res 33: 3487-3494, 2013

[109] Peereboom DM, Ahluwalia MS, Ye X, et al: Phase II and pharmacokinetic study of erlotinib and sorafenib for patients with progressive or recurrent glioblastoma multiforme. Neuro Oncol. 15(4): 490-6, 2013

[110] de Groot JF, Lamborn KR, Chang SM, et al. Phase II study of aflibercept in recurrent malignant glioma: a North American Brain Tumor Consortium study. J Clin Oncol 29: 2689-95, 2011

[111] Holash J, Davis S, Papadopoulos N, et al: VEGF-Trap: a VEGF blocker with potent antitumor effects. Proc Natl Acad Sci USA 99:11393-8, 2002

[112] Kesari S, Schiff D, Henson JW, et al: Phase II study of temozolomide, thalidomide, and celecoxib for newly diagnosed glioblastoma in adults. Neuro Oncol 10:300-8, 2008

[113] Chang SM, Lamborn KR, Malec M, et al: Phase II study of temozolomide and thalidomide with radiation therapy for newly diagnosed glioblastoma multiforme. Int $\mathrm{J}$ Radiat Oncol Biol Phys 60:353-7, 2004

[114] Reardon DA, Fink KL, Mikkelsen T, et al: Randomized phase II study of cilengitide, an integrin-targeting arginine-glycine-aspartic acid peptide, in recurrent glioblastoma multiforme. J Clin Oncol 26: 5610-7, 2008

[115] Stupp R, Hegi ME, Neyns B, et al: Phase I/IIa study of cilengitide and temozolomide with concomitant radiotherapy followed by cilengitide and temozolomide maintenance therapy in patients with newly diagnosed glioblastoma. J Clin Oncol 28:2712, 2010

[116] Stupp R, Hegi ME, Gorlia T, et al: Cilengitide combined with standard treatment for patients with newly diagnosed glioblastoma with methylatedMGMTpromoter (CENTRIC
EORTC 26071-22072 study): a multicentre, randomised, open-label, phase 3 trial. Lancet Oncol. 15:1100-8, 2014

[117] Downward J: Targeting RAS signalling pathways in cancer therapy. Nat Rev Cancer. 3:11-22, 2003

[118] Polivka J, Rohan V, Topolcan O and Ferda J: New molecularly targeted therapies for glioblastoma multiforme. Anticancer Res 32:2935-2946, 2012

[119] Feldkamp MM, Lau N, Guha A: Growth inhibition of astrocytoma cells by farnesyl transferase inhibitors is mediated by a combination of anti-proliferative, pro-apoptotic and antiangiogenic effects. Oncogene 18:7514-7526, 1999

[120] Cloughesy TF, Wen PY, Robins HI, et al: Phase II trial of tipifarnib in patients with recurrent malignant glioma either receiving or not receiving enzyme- inducing antiepileptic drugs: a North American Brain Tumor Consortium Study. J Clin Oncol 24:3651-3656, 2006

[121] de Boüard S, Herlin P, Christensen JG, et al: Antiangiogenic and anti-invasive effects of sunitinib on experimental human glioblastoma. Neuro Oncol 9:412-423, 2007

[122] Schmelzle T, Hall MN: TOR, a central controller of cell growth. Cell 103:253-262, 2000

[123] Wang SI, Puc J, Li J, et al: Somatic mutations of PTEN in glioblastoma multiforme. Cancer Res 57:4183-4186, 1997

[124] Galanis E, Buckner JC, Maurer MJ, et al: Phase II trial of temsirolimus (CCI-779) in recurrent glioblastoma multiforme: a North Central Cancer Treatmen Group Study. J Clin Oncol 23:5294-5304, 2005

[125] Hainsworth JD, Shih KC, Shepard GC, Tillinghast GW, Brinker BT, Spigel DR: Phase II study of concurrent radiation therapy, temozolomide, and bevacizumab followed by bevacizumab/ everolimus as first-line treatment for patients with glioblastoma. Clin Adv Hematol Oncol 10:240-246, 2012

[126] Millward MJ, Cantwell BM, Lien EA, et al: Intermittent high-dose tamoxifen as a potential modifier of multidrug resistance. Eur J Cancer 28A:805-810, 1992

[127] Montgomery RB, Moscatello DK, Wong AJ, et al: Differential modulation of mitogen-activated protein (MAP) kinase/extracellular signal-related kinase kinase and MAP kinase activities by a mutant epidermal growth factor receptor. J Biol Chem 270:30562-30566, 1995

[128] Couldwell WT, Hinton DR, Surnock AA, et al: Treatment of recurrent malignant gliomas with chronic oral high-dose tamoxifen. Clin Cancer Res 2:619-622, 1996

[129] Brandes AA, Ermani M, Turazzi S, et al: Procarbazine and high-dose tamoxifen as a secondline regimen in recurrent high-grade gliomas: a phase II study. J Clin Oncol 17:645-650, 1999

[130] Di Cristofori A, Carrabba G, Lanfranchi G, et al: Continuous tamoxifen and dose-dense temozolomide in recurrent glioblastoma. Anticancer Res 33: 3383-3389, 2013

[131] Wick W, Puduvalli VK, Chamberlain MC, et al: Phase III study of enzastaurin compared with lomustine in the treatment of recurrent intracranial glioblastoma. J Clin Oncol 
28:1168-1174, 2010

[132] Okura H, Smith C A and Rutka J T: Gene therapy for malignant glioma. Molecular and Cellular Therapies 2:21, 2014
[133] Uniyal M J, Labagnara M, Friedman M, et al: Glioblastoma: Molecular Pathways, Stem Cells and Therapeutic Targets. Cancers 7: 538-555, 2015 